\title{
Terapia ocupacional, cultura y diversidad ${ }^{1}$
}

\author{
Salvador Simó Algado \\ Universitat de Vic - Universitat Central de Catalunya, Vic, Espanha.
}

\begin{abstract}
Resumem: El comportamiento ocupacional siempre es un comportamiento medioambiental, ya que la ocupación es el diálogo del ser humano con su medio ambiente. Es por ello que la cultura, dimensión caudal del medioambiente, es clave en nuestra profesión. Este artículo de reflexión parte de investigaciones previas y de una nueva revisión de la literatura científica sobre los distintos significados asociados a la cultura. En el marco contemporáneo se está produciendo una globalización que implica la imposición del American way of life a escala planetaria. Ante el etnocentrismo y el racismo se propone una hermenéutica diatópica que apuesta por la diversidad y por la construcción de una ciudadanía cosmopolita. El artículo concluye reflexionando sobre estrategias que podemos implementar desde la terapia ocupacional para desarrollar terapias ocupacionales desde una praxis culturalmente segura. Ante la americanización se impone desarrollar una arqueología de los significados desde una terapia ocupacional transcultural. La terapia ocupacional debe recuperar su activismo social y político en post de la construcción de una sociedad basada en los derechos humanos y la sostenibilidad. La cultura y la diversidad son elementos claves en este proceso.
\end{abstract}

Palabras clave: Participación Ciudadana, Derechos Humanos, Globalización, Etnocentrismo, Multiculturalismo.

\section{Terapia ocupacional, cultura e diversidade}

Resumo: O comportamento ocupacional sempre é um comportamento meio ambiental, já que a ocupação é o diálogo do ser humano com seu meio ambiente. É por ele que a cultura, dimensão central do meio ambiente, torna-se chave para a terapia ocupacional. Este artigo é baseado em pesquisa prévia e uma nova revisão da literatura científica sobre os vários significados associados à cultura. Na contemporaneidade está sendo produzida uma globalização que implicada na imposição de um 'american way of life' (forma americana de vida) em escala planetária. Contra o etnocentrismo e o racismo se propõe uma hermenêutica diatópica que aposta na diversidade e na construção de uma cidadania cosmopolita. O artigo finaliza refletindo sobre as estratégias que podemos implementar a partir da terapia ocupacional para desenvolver terapias ocupacionais com práxis culturalmente seguras. Diante da americanização, se faz necessário desenvolver uma arqueologia de significados de uma terapia ocupacional transcultural. A categoria profissional deve recuperar seu ativismo social e político na direção da construção de uma sociedade inclusiva e sustentável, com base nos direitos humanos e sustentabilidade. Cultura e diversidade são elementos-chave neste processo.

Palavras-chave: Direitos Humanos, Participação Cidadã, Globalização, Etnocentrismo, Multiculturalismo.

\section{Occupational therapy, culture and diversity}

\begin{abstract}
Occupational behaviour is always an environmental behaviour because the occupation is the dialogue between human beings and their environment. Culture is key dimension in our profession. This article is based on previous research and a new review of the scientific literature on the various meanings associated with culture. In the contemporary context the globalization involves the imposition of the American way of life on a planetary
\end{abstract}

Autor para la correspondencia: Salvador Simó Algado, Grupo de investigación Salud Mental e Innovación Social, Facultad de Ciencias de la Salud y del Bienestar, Universidad de Vic., Sagrada Familia, 708500 Vic, Espanha, e-mail: salvador.simo@uvic.cat Recibido Mayo 30, 2015; Revisión Ago 10, 2015; Aceptado Ago 30, 2015. 
scale. In front of ethnocentrism and racism a diatopical hermeneutics is proposed. A cosmopolitan citizenship can be constructed supporting diversity. The article concludes by reflecting on strategies that can be implemented from occupational therapy to develop a culturally safe occupational therapy. An archaeology of meaning must be developed in front of the Americanization. Occupational therapy needs to develop a powerful political activism in order to build an inclusive society based on human rights and sustainability. Culture and diversity are key elements in this process.

Keywords: Human Rights, Citizen participation, Globalization, Ethnocentrism, Multiculturalism.

\section{La cultura}

Seremos compatriotas de todos lo que tengan voluntad de justicia y voluntad de belleza, hayan nacido donde hayan nacido, sin que importen ni un momento las fronteras del mapa (GALEANO, 2015, s/p.).

El comportamiento ocupacional siempre es un comportamiento medioambiental, ya que la ocupación es el diálogo entre el ser humano con su medio ambiente. Es por ello que la cultura es una dimensión clave en nuestra profesión. Cultura proviene del latín cultura y según la Real Academia Española (REAL..., 2015a) significa: a) El conjunto de modos de vida, costumbres, conocimientos y grado de desarrollo artístico, científico e industrial, de una época o grupo social. b) Las nociones populares incluyen en ocasiones aspectos de raza e identidad étnica, así como lo que ha venido a llamarse alta cultura: moda y formas artísticas.

Según Bauman (2009) existen dos formas principales de comprender la cultura. Una generó la idea de cultura como la actividad del espíritu libre. Es la sede de la creatividad, de invención, de la autocrítica y de la autotrascendencia. La otra plantea la cultura como un instrumento de continuidad, al servicio del orden social. La primera concepción entiende la cultura como capacidad de resistirse a las normas, de erigirse por encima del ordinario. Es la poiesis, arte, creación ab nibilo al estilo divino. Significa aquello que distinguía a los espíritus más atrevidos. Para la segunda significa regularidad y modelo. La cultura es un agregado o sistema coherente de presiones apoyadas sobre sanciones, de valores y normas interiorizados, que garantiza la preservación de la tradición. Las dos culturas se erigen una frente a la otra: la una niega lo que la otra proclama. Así Bauman (2009) concluye que la cultura es un agente del desorden tanto como un instrumento del orden, un elemento sometido a los rigores del envejecimiento como un ente atemporal. Es a la vez un espacio de creatividad y un marco de regulación normativa. La cultura es un complejo concepto.
No podemos obviar las reflexiones de Adorno (2003) sobre la cultura de masas, una cultura que no emerge de las masas sino que está dirigida a las masas. La cultura se convierte en un elemento domesticador y de control social, un mero objeto de consumo propio de una sociedad capitalista.

Dyck (1998) explica la cultura como un sistema compartido de significados que abarca ideas, conceptos y conocimientos, e incluye creencias, valores y normas que forman los estándares y las reglas de comportamiento emprendidas por las personas en su vida cotidiana. Kymlicka (1996, p. 112) define la cultura de una sociedad como

[...] aquello que proporciona a sus miembros unas formas de vida significativas a través de todo el abanico de actividades humanas, incluyendo la vida social, educativa, recreativa y económica, acercando las esferas pública y privada. Las culturas tienen a concentrarse territorialmente y se basan en una lengua compartida.

\section{Globalización y genocidio cultural}

La sociedad contemporánea está marcada por globalización. Si bien no se ha producido una globalización de los derechos humanos si que se ha producido una globalización de los mercados económicos. Así los gobiernos eliminan las barreras de protección de sus propios mercados. Paradójicamente se garantiza la libre circulación de los capitales, pero no la circulación de las personas. A nivel laboral, está implicando la precarización del mercado laboral global. Se ha producido una igualación de dichas condiciones laborales a la baja, basada en el empeoramiento de las condiciones de los países donde los trabajadores gozaban de mayores garantías y derechos laborales. Beck (2000) habla de la brasileñización del mercado laboral europeo.

A nivel cultural nos gustaría pensar en la globalización como un proceso de intercambio y enriquecimiento mutuo cultural. Pero ha implicado la exportación del American way of life a escala planetaria. Así podemos hablar de la generación 
$M T V$ o de la McDonalización del mundo. Lo más preocupante es que este proceso sigue a un genocidio cultural iniciado hace siglos. No podemos olvidar que al cabo de 150 ańos del inicio de la colonización europea de América, ya habían desaparecido más de un $90 \%$ de las comunidades originarias indígenas. Compte-Sponville (2003) define genocidio como el exterminio de un pueblo, que constituye un crimen contra la Humanidad. La existencia de los pueblos indígenas está amenazada. El pintor indígena Gauyasamín fue capaz de plasmar en obras como Rios de sangre (1976) este genocidio sobre el lienzo, al que cantó Neruda:

Estos dolores / se exprimirán como pañuelos / hasta estrujar tantas lágrimas vertidas / en socavones del desierto, en tumbas / en escalones del martirio humano (GUAYASAMÍN; NERUDA, 2006, p. 115).

Davis (2001) expone como alrededor de 300 millones de personas mantienen una fuerte identidad como miembros de una cultura indígena. No hay una mayor medida que refleje esta crisis cultural que la pérdida de lenguas. De las 10.000 que han existido, hoy 6.000 todavía se hablan, pero solo 600 lenguas son consideradas estables. Desde un punto de vista económico su pérdida parece superflua. Pero una lengua es un reflejo del espíritu humano, el filtro a través del cual el alma de cada cultura particular se relaciona con el mundo. Un lenguaje es un ser vivo, divino y misterioso, un ecosistema completo de ideas y de instituciones, una cascada de pensamiento, un bosque milenario de la mente. En peligro se encuentra la expresión espiritual, intelectual y artística con toda la complejidad y la diversidad de la experiencia humana. En Canadá, en la tierra de los Mikmak, los árboles son denominados por el sonido que los vientos perpetuos hacen al mecer las ramas en el otoño, una hora después del crepúsculo. ¿Cuál es el valor de los vínculos familiares que mitigan la pobreza y protegen a las personas de la soledad? ¿Cuál es el valor de las diversas instituciones sobre el cosmos, los senderos del espíritu, el significado de la práctica de fe? ¿Cuál es el valor de una práctica ritual que protege un río o un bosque?

\section{Etnocentrismo}

Negro, nombre dado a una variedad de la especie humana... Algunos de sus vicios más notorios son ociosidad, venganza, descaro, robo, mentira, mentira, libertinaje, suciedad... Desconocen la compasión y constituyen un terrible ejemplo de corrupción (ENCICLOPEDIA..., 1798, p. 794).
¿Cómo podemos permanecer impasibles ante este genocidio cultural? Occidente siempre se ha relacionado con las demás culturas desde una posición de descubridor. Según Sousa (2005), el descubrimiento es una relación de poder y de saber, donde es descubridor quien tiene mayor poder y saber así como la capacidad de declarar al otro como descubierto, transformando la reciprocidad del descubrimiento en apropiación del descubierto. El segundo milenio fue el milenio de los descubrimientos. El descubrimiento establece la inferioridad del otro y la legítima. Lo que se descubre está lejos, abajo y en los márgenes. La producción de la inferioridad es crucial y recurre a múltiples estrategias como la guerra, la esclavitud, el genocidio, el racismo, la transformación del otro en objeto o en recurso natural, mecanismos de imposición económica (tributos, colonialismo, neocolonialismo y a la fin globalización neoliberal), de imposición política (cruzadas, imperio, estado colonial, dictadura) y de imposición cultural (epistemicidio, misiones, asimilación y finalmente industrias culturales y cultura de masas).

El Otro para Occidente asumió tres formas: Oriente, el salvaje y la naturaleza. Oriente es el espacio de alteridad. Hasta el siglo XV Occidente es la periferia de un sistema mundo con su centro localizado en Oriente, la civilización temida y admirada a la vez. El salvaje es el espacio de la inferioridad, diferencia incapaz de constituirse en alteridad. Su valor es el de utilidad, el de recurso. La incondicionalidad de los fines justifica la esclavitud, el genocidio, la apropiación o la asimilación. La naturaleza es el tercer descubrimiento del segundo milenio. Es el lugar de la exterioridad, lo que no pertenece. La única finalidad es el dominio y el control, es un mero recurso natural.

El descubrimiento imperial no reconoce derechos de igualdad o dignidad. Oriente es el enemigo, el salvaje es inferior y la naturaleza es un recurso. El etnocentrismo es una visión de las cosas en que un grupo se erige como el centro de todo, los demás son clasificados y tasados con referencia a dicho centro (SUMMER, 1906). Como afirma Gergen (1996) la cultura occidental no ha querido ver a las otras culturas como superiores o iguales.

Con Sousa (2005, p. 150) nos preguntamos:

¿Son posibles los redescubrimientos? ¿Es posible sustituir Oriente por una convivencia multicultural? ¿Es posible sustituir el salvaje por una igualdad en la diferencia y por la autodeterminación? ¿Es posible sustituir la naturaleza por una humanidad que la incluya? 


\section{La diversidad: El encuentro con el Otro}

La diversidad cultural es una fuerza motriz de desarrollo económico, intelectual, afectivo, moral y espiritual (UNESCO, 2002, s/p).

Diversidad proviene del latín diversittas, -ätis. Según la Real Academia Española (2015b) significa: 1. Variedad, desemejanza, diferencia; 2 . Abundancia, gran cantidad de varias cosas distintas. La diversidad es un rasgo fundamental la humanidad, es nuestra riqueza y patrimonio.

El ser humano implica el encuentro con el Otro. Para Kapuscinski (2007) este encuentro supone una incógnita siempre presente y renovada, un planteamiento tan antiguo que casi podría clasificarse de eterno. El Otro parece llevar en su interior a dos personas. Una es un ser como todos nosotros, con sus alegrías y sus tristezas. El segundo ser es portador de unos rasgos raciales, de una cultura, de una ideología.

Kapuscinski (2007, p. 27) se pregunta: ¿Quién será ese nuevo Otro? ¿Cómo transcurrirá nuestro encuentro? ¿Qué nos diremos? ¿En qué lengua? ¿Sabremos escucharnos? ¿Sabremos entre los dos seguir aquello que habla de nuestra capacidad de alegría y admiración, se dirige al sentimiento del misterio que rodea nuestras vidas, a nuestro sentido de la piedad, de la belleza y del dolor, al sentimiento que nos vincula con la creación; y a la convicción de la solidaridad que une a la soledad de innumerables corazones; a esa solidaridad en los sueńos, en el placer, en la tristeza, en los anhelos, en las ilusiones, en la esperanza, en el temor, que relaciona cada hombre con su prójimo y mancomuna toda la humanidad, los muertos con los vivos, y los vivos con aquellos que aún han de nacer?

La cultura contemporánea se debate entre una globalización uniformadora y un etnonacionalismo diferenciador. Esta tensión crea una confusión de identidades que sumado a la crisis hace atractivos los cantos de sirena de los discursos racistas. Lo más fácil es culpar al Otro de todos los males. El encuentro es inevitable, por factores como la revolución electrónica, el desarrollo de comunicaciones y las terribles desigualdades del mundo. La pregunta es: ¿`cómo afrontaremos lo inevitable? ¿Desde la civilización o desde la barbarie?

Sousa (2005) propone una hermenéutica diatópica basada en la idea de que todas las culturas son incompletas y que los tópoi o creencias fuertes de argumentación de cada cultura también están incompletos. Los tópoi hacen posible la creación de argumentos y su intercambio. Estos producen la ilusión de totalidad. El objetivo de la hermenéutica diatópica es crear conciencia sobre la incompletud recíproca de las culturas a través del diálogo intercultural. Uno de los tópoi de nuestra cultura es la creencia en nuestra independencia, acompañada del cada uno para sí y si es necesario contra sus semejantes, en un rudo individualismo basado en la supervivencia del más apto. Esta idea supuestamente evoluciona de las leyes de la naturaleza. Pero el mundo de la biología, junto a la supervivencia del más fuerte, nos ofrece muchos ejemplos de cooperación y de sinergias entre especies (PIGEM, 1994). Esta creencia en la independencia contrasta con la visión propia de las culturas asiáticas y de los pueblos indígenas, basada en la interdependencia entre todos los seres humanos y con el medio natural.

\section{Una ciudadanía inclusiva y cosmopolita}

Se impone el instituir una ciudadanía cosmopolita enraizada en lo local. Por una parte se justifica desde un imperativo moral, ya que hay medios suficientes para que nadie vea insatisfechas sus necesidades básicas. Sólo la solidaridad es una actitud éticamente acertada para acabar con la injusta exclusión y hacer partícipes de los bienes de la Tierra, materiales e inmateriales a todas las personas (CORTINA, 2005). Pero también es un imperativo de supervivencia, ante los desafíos globales que enfrentamos sólo cabe una respuesta global, porque la humanidad es un barco que avanza por las aguas tempestuosas de la historia. Un mundo global es un mundo en que el desiderátum de la responsabilidad moral y los intereses de supervivencia se funden.

El cosmopolitismo nos remite a una ciudad sin murallas. Debemos distinguir entre un cosmopolitismo cultural, económico y político moral. El cultural implica un distanciamiento de los marcos nacionales y una actitud de apertura y comunicación, sin tener en cuenta las fronteras, pero sin abolirlas. El económico es teorizado por el neoliberalismo y empujado por la globalización de los mercados. El moral y político establece una serie de derechos y obligaciones morales entre todos los seres humanos (PEÑA, 2010). Kant (1946) planteaba la idea de una historia universal en sentido cosmopolita, la unificación perfecta de la especie humana a través de una ciudadanía común, como el supremo designio 
de la naturaleza, ya que habitamos en una esfera que impide la dispersión infinita.

En la tensión entre lo local y lo global, debemos encontrar el equilibrio en lo glocal, un cosmopolitismo desde el enraizamiento en lo local (ETXEBERRIA, 2003), desde ciudadanías multiculturales porque los seres humanos somos humanos en culturas particulares (GEERTZ, 1996). El universalismo debe ser altamente sensible a la diferencia, porque la homogeneización mata la vida, sólo la diversidad de las actividades humanas la vivifican (CORTINA, 2008). El solidario hacerse cargo del otro se refiere al flexible nos-otros de una comunidad que amplía cada vez más sus porosos límites. Esta comunidad moral se construye sobre la base de la eliminación de la discriminación y del sufrimiento (HABERMAS, 1999).

La ciudadanía multicultural, sensible a las diferencias étnicas, surge del hecho de que dentro de la democracia las mayorías pueden obviar los derechos de las minorías. Hay diferencias culturales que sus portadores quieren mantener; y por ello solicitan cierta protección en el ámbito público, por lo que sin negar las políticas de igualdad reclaman a su vez políticas de la diferencia, que reconozcan junto a los derechos humanos individuales los derechos diferenciales de los grupos en función de su identidad (ETXEBERRIA, 2003). Surge un ciudadano global, cosmopolita, que demuestra interés en conocer y reflexionar críticamente sobre los problemas mundiales y sobre cómo interactúan en nuestras vidas y en la de los otros, con conciencia de la interdependencia mutua. Un ciudadano arraigado en lo local que se sitúa en el mundo a través de formas e identidades complejas. De ahí su respeto a la diversidad y su indignación frente a la exclusión. Es consciente de sus derechos y obligaciones y se responsabiliza de sus acciones como ciudadano planetario. Es una persona responsable, crítica, que no sólo exige sus derechos y cumple sus obligaciones, sino que, además, también lucha por un mundo mejor para todos (PAZ ABRIL, 2007).

\section{Reflexiones finales. Terapia ocupacional, diversidad y cultura}

De lo dicho hasta aquí podemos hacer algunas reflexiones:

\subsection{Terapias ocupacionales desde un paradigma crítico}

Debemos desarrollar una terapia ocupacional desde un paradigma crítico cuando estamos vivenciando la desaparición de la diversidad cultural. Como dice Dussel (2006) el sufrimiento de las víctimas es nuestro punto de arranque fuerte. El paradigma crítico está basado en la creencia en la dignidad humana y en nuestra capacidad de mejorar la realidad presente. Ante la situación de sufrimiento evitable debemos recobrar el sentido de la cultura como capacidad de resistirse a las normas, de transformar la injusticia establecida, de poiesis y arte, en la co-creación de una sociedad más justa e inclusiva, basada en los derechos humanos y la sostenibilidad. No se trata tan solo de conceptos para incorporar a declaraciones, como cultura o diversidad, sino de reivindicar y defender desde un verdadero activismo nuestro patrimonio cultural humano.

La terapia ocupacional hasta la fecha ha sido dominada por el punto de vista anglosajón, ya que la profesión tiene su origen en una sociedad anglosajona blanca de clase media. De la misma forma que podemos hablar de un colonialismo territorial podemos hablar de un colonialismo académico o teórico. Este etnocentrismo se manifiesta en realidades como que el inglés es impuesto como el único idioma viable para la comunicación científica. Por tanto es fundamental desarrollar una hermenéutica diatópica à la Sousa dentro de la profesión, desarrollando así un re-descubrimiento para reconocer y poner en valor las terapias ocupacionales desarrolladas desde los contextos no anglosajones.

Esta hermenéutica diatópica debe ir más allá de la propia profesión. Es fundamental conocer y aprender de profesionales fuera del ámbito de la terapia ocupacional. Más allá de las ciencias de la salud debemos interactuar con otros profesionales incorporando conocimientos de la antropología, la filosofía, la economía, la ecología, la empresa, las nuevas tecnologías, etc.

En la Tabla 1 el lector encontrará dos ejemplos de producción de conocimiento desde el Sur.

\subsection{Una praxis de la terapia ocupacional culturalmente segura}

Es preciso desarrollar una praxis de la terapia ocupacional culturalmente segura. No es posible exportar modelos teóricos y teorías basadas en cosmovisiones diferentes. Por ejemplo, no es posible exportar el modelo de la ocupación humana a Japón cuando en la cultura japonesa no existe el concepto de causalidad personal (IWAMA; SIMÓ ALGADO, 2008a). Una terapia ocupacional fuera del contexto cultural puede convertirse en una profesión irrelevante, o en el peor de los casos en un agente de opresión que colonizara e 
Tabla 1. Terapia ocupacionales desde el Sur.

En este sentido podemos destacar la producción de proyectos editoriales como Terapias ocupacionales desde el Sur: Derechos humanos, ciudadan y participación (SIMÓ ALGADO et al., 2016). Se trata de un libro con la participación de Brasil, España, Chile, Colombia, Argentina y Venezuela. Este proyecto pone en valor y en comunicación saberes y praxis de la terapia ocupacional desde una epistemología del Sur. Pone de manifiesto una comprensión diferente de la terapia ocupacional, donde cobran relevancia conceptos como los derechos humanos, la ciudadanía, la participación, la terapia ocupacional eco-social o la rehabilitación basada en la comunidad.

Otro texto que debemos mencionar es Questões contemporânea da Terapia Ocupacional na América do Sul (SANTOS; DONATTI, 2014).

incluso marginara a las personas. Para introducir la terapia ocupacional en las vidas de otras gentes de forma segura y equitativa, debemos entender que la cultura y la diversidad son conceptos claves de nuestras intervenciones (IWAMA; SIMÓ ALGADO, 2008b).

En la Tabla 2 el lector encontrará un ejemplo sobre la importancia de la cultura en una intervención desarrollada desde terapia ocupacional con refugiados indígenas mayas.

\subsection{Una arqueología de los significados}

La terapia ocupacional se basa en el desarrollo de ocupaciones significativas. Pero debemos ser críticos a la hora de asumir el significado asociado a las distintas ocupaciones, por ejemplo, cuando el $90 \%$ de los adolescentes americanos identifica el shopping como su actividad de ocio favorita (SUZUKI, 2002). Ante el proceso Americanización es fundamental desarrollar una arqueología de los significados. Afirmaba Marx (1988) que las ideas dominantes en cada sociedad son las propias de sus clases dominantes. El control de la población en sociedades como la norteamericana se realiza de forma más sutil, a través de adoctrinar de los jóvenes, en las escuelas y las universidades (CHOMSKY, 2003). Son ya clásicas las reflexiones de Freire (2009) sobre la educación bancaria. El sistema neoliberal preponderante precisa de consumidores, que no de ciudadanos, para seguir existiendo. Por lo tanto educa en la religión del consumo a partir de una ética hedonista. Convierte así el homo faber en un triste homo consumens.

Es por ello fundamental cuando buscamos ocupaciones significativas con la persona, grupo o comunidad, desarrollar una arqueología de los significados, que consiste en indagar sobre los significados que atribuyen a las ocupaciones y su procedencia. Así podemos comprobar si éstas realmente responden a su esencia y personalidad, si van a permitir su desarrollo humano, potenciando su bienestar y su participación social, o simplemente han sido impuestas por el sistema dominante y son un elemento de alienación y domesticación. A partir de aquí es posible explorar nuevas ocupaciones asociadas a nuevos valores y significados que hasta el momento no se han desarrollado.

En la Tabla 3 se ejemplifica a partir de un caso la necesidad de desarrollar una arqueología del significado.

\subsection{Una terapia ocupacional transcultural}

Un aspecto básico es desarrollar una terapia ocupacional transcultural (SIMÓ ALGADO; BURGMAN, 2006), esta terapia no sólo reconoce la belleza inmanente a toda cultura si no que busca introducir ocupaciones culturalmente significativas en cada intervención.

Latinoamérica puede y debe ser un escenario del desarrollo de una terapia ocupacional transcultural, ya que todavía conserva una gran riqueza cultural. Como terapeutas ocupacionales debemos tomar consciencia de que la diversidad cultural de la humanidad sigue amenazada. Se impone la necesidad de trabajar con estas comunidades amenazadas, desde la conciencia de la riqueza que suponen y desde un proceso de aprendizaje mutuo. La globalización implica que estas culturas están perdiendo sus formas ancestrales de ganarse la vida e impacta de forma drástica en sus patrones ocupacionales generando una disfunción ocupacional. Se abre ante nosotros un fascinante escenario de intervención en países como Brasil. Como terapeutas ocupacionales debemos celebrar las intervenciones desarrolladas por compañeras como Maria Daniela Macedo, Samiria Lima Costa o Elisabeth Lima en Brasil, sin olvidarnos del fascinante trabajo de Denise Barros con los Dogón en Mali; por citar unos ejemplos. Estas intervenciones nos inspiran como profesión y nos marcan el camino a seguir. 
Tabla 2. Una intervención con una comunidad maya.

En la intervención desarrollada por el primer autor (SIMÓ ALGADO; GREGORI; EGAN, 1997; SIMÓ ALGADO; CARDONA, 2006) con una comunidad de refugiados mayas que retornaban a Guatemala después de 14 años de exilio en México, fue fundamental el aprender de la cosmovisión maya. Con dicho fin estuvimos una semana aprendiendo con un sacerdote maya, o Ajij. Éste nos explicó los principios de la cosmovisión Maya, el sistema de creencias y significados que ordenan su existencia. Así aprendimos que en el universo coexisten el bien y el mal en una lucha eterna de la que nosotros formamos parte de la misma forma que hicieron nuestros antepasados. Esta creencia nos ayudó a darle un significado a las masacres sufridas por la comunidad.

Aprendimos la extrema importancia de desarrollar ocupaciones de cuidado de la naturaleza ya que creen firmemente en la interdependencia del ser humano y la tierra. "Si la tierra enferma del ser humano enfermará sin esperanza". Una de las creencias de los mayas es la existencia del nagual, al nacer todo ser humano queda relacionado con un elemento de la naturaleza compartiendo un destino común. Es una potente metáfora de la dependencia del ser humano de la Tierra. Estas lecciones han influenciado el trabajo sobre ecología ocupacional del primer autor (SIMÓ ALGADO, 2012; SIMÓ ALGADO; TOWNSEND, 2015).

Tabla 3. Patricia.

Patricia tiene 17 años, es esbelta y atractiva. Procede de una familia desestructurada. Carece de estudios
reglados más allá de la educación básica. Actualmente sus únicos intereses son su imagen física, la ropa y los
perfumes de marca y poder salir de fiesta. Sus valores giran entorno al hedonismo, al consumismo y la imagen
personal. Ha empezado a prostituirse hace unos meses.
El terapeuta ocupacional que trabaja con Patricia busca alternativas profesionales. Le ofrece a Patricia que
puede formarse como peluquera, cobrando una beca salario de $400 €$ mensuales (el salario mínimo en España
está en torno a los $640 €$ ). Pero Patricia gana mucho más dinero en un fin de semana. De hecho piensa que no
hay nada malo en prostituirse, ya que la prostitución le da acceso a las cosas que actualmente valora.
Este caso ilustra la necesidad de explorar unos nuevos valores que se traduzcan en nuevos patrones
ocupacionales.

Tabla 4. Poemas culturalmente significativos.

Durante la intervención con los niños supervivientes de la guerra en Kosovo (SIMÓ ALGADO; BURGMAN,
2006), el objetivo era facilitar la expresión de las vivencias de guerra para evitar el desarrollo de un trauma
psicológico posterior. Para facilitar dicha expresión se incorporaron poemas que la propia comunidad había
creado que narraban las masacres acaecidas en Kosovo. Estos poemas eran una forma social de expresar el
dolor por los muertos y los desaparecidos. Era notorio ver como niños que eran pocos expresivos cambiaban
radicalmente a recitar estos poemas. Los poemas demostraron un gran potencial terapéutico para facilitar la
expresión de las vivencias traumática.

En la Tabla 4 el lector encontrará un ejemplo de cómo se introdujeron poemas culturalmente significativos, desde una terapia ocupacional transcultural, en una intervención con niños supervivientes de guerra.

\subsection{Ciudadanía y terapia ocupacional}

Como terapeutas ocupacionales debemos trabajar en pos de una ciudadanía cosmopolita y multicultural. Nuestro objetivo es la creación de comunidades saludables, inclusivas y sostenibles, donde toda persona sea reconocida como ciudadana de pleno derecho a pesar de cualquier disfunción ocupacional, debida a motivos físicos, psicológicos, sociales o medioambientales. Como terapeutas ocupacionales debemos incorporar el lenguaje de los derechos humanos y la sostenibilidad en nuestras intervenciones (SIMÓ ALGADO, 2012). Es fundamental concientizar à la Freire a las personas y comunidades sobre sus derechos y deberes de ciudadanía. Es fundamental desarrollar un trabajo de reivindicación de los derechos humanos (GUAJARDO; SIMÓ ALGADO, 2010) desde un potente activismo social y político, desarrollando una ciudadanía cosmopolita y multicultural.

En la Tabla 5 el lector encontrará dos ejemplos relacionados con los derechos humanos. Uno explica como Brasil es escenario de un fascinante trabajo en pro de los derechos humanos a partir del arte y la cultura. Por otra parte, muestra un ejemplo de un trabajo de concientización y reivindicación de los derechos humanos, desarrollado con los niños en Barcelona. 
Tabla 5. Terapia Ocupacional, derechos humanos y ciudadanía.

Proyecto editorial: Direitos humanos para diversidade: construindo espaços de arte, cultura e educação. Desde hace años está emergiendo una poderosa terapia ocupacional desde el gran Sur. Sin duda Brasil es uno de los epicentros de esta epifanía de conocimiento, un conocimiento vivo, ya que lejos de quedarse en la abstracción teórica contribuye a la transformación social. Un excelente ejemplo es el trabajo que los terapeutas ocupacionales están desarrollando desde el arte y la cultura como medio de transformación social y de promoción de los derechos humanos. Un excelente ejemplo que refleja esta realidad es el libro Direitos humanos para diversidade liderado por Carla Silva (2014) desde la Universidad de San Carlos.

\section{Proyecto Renacimiento: Tengo derechos, tengo deberes}

Este proyecto se ha diseñado por el primer autor conjuntamente con las escuelas de la ciudad de Vic (España), para presentarlo a los presupuestos participativos. El proyecto se estructura en cuatro ejes: Bienestar, Arte, Sostenibilidad, Ciudadanía. En dicho eje se pretende desarrollar una labor de concientización con los niños y sus familiares en relación a sus derechos humanos, desde la dialéctica derechos/deberes. Por un lado los niños asumirán sus deberes y se comprometerán en la mejora de la ciudad a partir de proyectos de aprendizaje servicio. Pero por otra parte se les concientizará de sus derechos y sobre cómo pueden reivindicarnos. Una de las acciones será la campaña Tengo derechos tengo deberes. A partir de videograbaciones los niños explicarán al resto de la ciudadanía lo que están haciendo como ciudadanos comprometidos. Pero también en estos videos denunciarán las situaciones de incumplimiento de sus derechos.

\section{Conclusión}

Cultura y diversidad son conceptos claves para la terapia ocupacional. Es preciso trascender la visión de la cultura como elemento de colonización, domesticación y de control social, incorporando la cultura como elemento de creación, de emancipación y de resistencia ante el orden establecido. Ante el predominio anglosajón en la profesión debemos defender la diversidad y desarrollar terapias ocupacionales, poniendo en valor el conocimiento y las praxis generadas desde el Sur. Se impone desarrollar una praxis culturalmente segura; una terapia ocupacional transcultural, introduciendo ocupaciones culturalmente significativas en nuestras intervenciones.

La cultura está relacionada con la creación de significados. Ante la imposición del Amercian way of life a escala planetaria, en el marco de una ética hedonista y consumista, precisamos desarrollar una arqueología de los significados, en la búsqueda de aquellas ocupaciones que sean realmente significativas para las personas, grupos y comunidades, para promover su crecimiento personal, su bienestar y su participación social.

Desde un activismo social y político de la terapia ocupacional, la cultura es un poderoso elemento de resistencia ante la deshumanización y la mercantilización del mundo, un instrumento de co-creación de una sociedad más justa e inclusiva, basada en los derechos humanos y la sostenibilidad. Una sociedad que valora la diversidad, donde todas las personas puedan participar como miembros de pleno derecho desde una ciudadanía cosmopolita y multicultural.

\section{Referencias}

ADORNO, T. Obra completa. Madrid: Akal, 2003.

BAUMAN, Z. La cultura como praxis. Barcelona: Paidós, 2009.

BECK, U. Un nuevo mundo feliz. Barcelona: Paidós, 2000.

CHOMSKY, N. Hegemony or survival. America's quest for global dominance. New York: Metropolitan Books, 2003.

COMPTE-SPONVILLE, A. Diccionario filosófico. Barcelona: Paidós Contextos, 2003.

CORTINA, A. Ciudadanos del mundo. Madrid: Alianza Editorial, 2005.

CORTINA, A. Ética aplicada y democracia radical. Madrid: Tecnos, 2008.

DAVIS, W. Light at the edge of the world. Toronto: Douglas McIntyre, 2001.

DUSSEL, E. Ética de la liberación en la sociedad de la globalización y la exclusión. Madrid: Editorial Trotta, 2006.

DYCK, I. Multicultural society. En: JONES, D. et al. Sociology and occupational therapy: an integrated approach. Edinburgh: Churchill Livingstone, 1998. p. 67-80.

ENCICLOPEDIA BRITANNICA. Filadelfia: Enciclopedia Britannica, 1798.

ETXEBERRIA, X. La ciudadanía de la interculturalidad. En: VIGIL, N.; ZARIQUIEY, R. Ciudadanias inconclusas. Lima: Pontificia Universidad Católica del Perú, 2003. p. 91-110.

FREIRE, P. Pedagogía del oprimido. Madrid: Siglo XXI, 2009. 
GALEANO, E. El derecho al delirio. Entrevista concedida a TV3. Disponible: <https://youtu.be/sX0ZSdZLXtc>. Acceso: 10 abr. 2015.

GEERTZ, C. Los usos de la diversidad. Barcelona: Paidós, 1996.

GERGEN, K. Realidades y relaciones. Aproximaciones a la construcción social. Barcelona: Paidós, 1996.

GUAJARDO, A.; SIMO ALGADO, S. Una terapia ocupacional basada en los derechos humanos. Revista TOG, Galícia, v. 7, n. 12, p. 1-25, 2010.

GUAYASAMÍN, O.; NERUDA, P. América, mi hermano, mi sangre. Nueva York: Ocean Sur, 2006.

HABERMAS, J. La inclusión del otro. Barcelona: Paidós, 1999.

IWAMA, M. K.; SIMÓ ALGADO, S. El modelo kawa (Rio). Revista TOG, Galícia, v. 5, n. 8, p. 1-24, 2008 a.

IWAMA, M. K.; SIMÓ ALGADO, S. Aspectos de significado, cultura e inclusión en Terapia Ocupacional. Revista TOG, Galícia, v. 5, n. 8, p. 1-24, 2008b.

KANT, I. Lo bello y lo sublime. Madrid: Espasa Calpe, 1946.

KAPUSCINSKI, R. Encuentro con el otro. Barcelona: Editorial Anagrama, 2007.

KYMLICKA, W. Ciudadanía multicultural. Madrid: Paidós, 1996.

MARX, K. Textos cardinales. Barcelona: Ediciones Península. 1988.

PAZ ABRIL, D. Escuelas y educación para la ciudadania global. Barcelona: Intermon Oxfam, 2007.

PEÑA, J. La ciudad sin murallas. Mataró: El Viejo topo, 2010.

PIGEM, J. Nueva conciencia. Barcelona: Integral, 1994.

REAL ACADEMIA ESPAÑOLA - RAE. Definición de cultura. Madrid. Disponible: <http://lema.rae.es/ drae/?val=cultura $>$. Acceso: 10 mar. 2015a.

REAL ACADEMIA ESPAÑOLA - RAE. Definición de diversidad. Madrid. Disponible: <http://lema.rae.es/ drae/?val=diversidad $>$. Acceso em: 10 mar. 2015b.
SANTOS, W.; DONATTI, A. (Org.). Questões contemporânea da Terapia Ocupacional na América do Sul. Rio de Janeiro: CRV, 2014.

SILVA, C. R. (Org.). Direitos humanos para diversidade: construindo espaços de arte, cultura e educação. São Carlos: Editora São Jorge, 2014.

SIMÓ ALGADO, S. et al. Derechos humanos, ciudadan y participación. Santiago de Chile: USACH, 2016. En prensa.

SIMÓ ALGADO, S. Terapia ocupacional ecosocial: hacia una ecología ocupacional. Cadernos de Terapia Ocupacional da UFSCar, São Carlos, v. 20, n. 1, p. 7-16, 2012.

SIMÓ ALGADO, S.; BURGMAN, I. Intervención mediante terapia ocupacional con niños supervivientes de conflicto bélico. En: KRONENBERG, F; SIMÓ ALGADO, S.; POLLARD, N. Terapia Ocupacional sin fronteras: aprendiendo del espíritu de supervivientes. Madrid: Editorial Médica Panamericana, 2006. p. 2640 .

SIMÓ ALGADO, S.; CARDONA, C. E. El regreso del hombre de maíz: un proyecto de intervención con una comunidad maya de retornados guatemaltecos. En: KRONENBERG, F.; SIMÓ ALGADO, S.; POLLARD, N. Terapia Ocupacional sin Fronteras: aprendiendo del espíritu de supervivientes. Madrid: Editorial Médica Panamericana, 2006. p. 339-354.

SIMÓ ALGADO, S.; GREGORI, J. M. R.; EGAN, M. Spirituality in a refugee camp. Canadian Journal of $O c$ cupational Therapy, Ottawa, v. 64, n. 3, p. 88-94, 1997.

SIMÓ ALGADO, S.; TOWNSEND, E. A. Eco-social occupational therapy. British Journal of Occupational Therapy, London, v. 78, n. 3, p. 182-186, 2015.

SOUSA, B. El milenio huérfano. Ensayos para una nueva cultura política. Madrid: Editorial Trotta, 2005.

SUMMER, W. Folkways. New York: Ginn, 1906.

SUZUKI, D. The sacred balance. Toronto: Greystone Books, 2002.

UNESCO. Declaración universal sobre la diversidad cultural. San Borja, 2002. Disponible: <http://unesdoc. unesco.org/images/0012/001271/127162s.pdf>. Acceso em: 10 abr. 2015.

\section{Notas}

${ }^{1}$ Este material procede tanto de la pesquisa como de proyectos desarrollados durante su praxis profesional como terapeuta ocupacional. 